\title{
Caracterización social y económica de la agricultura tradicional de Carhuaz, Ancash
}

\author{
Social and economic characterization of \\ traditional farming of Carhuaz, Ancash
}

\section{Juan Barreto Rodríguez; Manuel Canto Sáenz ${ }^{2}$ Alberto Julca Otiniano²; Félix Camarena Mayta ${ }^{2}$}

\section{RESUMEN}

Objetivo: Determinar los sistemas de producción agropecuaria tradicional predominantes en la zona y las características socio económicas de la población de la Cordillera Negra en Carhuaz, Ancash. Materiales y métodos: Se aplicaron encuestas a una muestra representativa de 339 jefes de familia, de los cinco distritos involucrados: Yúngar, Anta, Marcará, Carhuaz y Ataquero, también entrevistas a tres líderes de la comunidad y cuatro alcaldes de los distritos en mención. Los datos obtenidos fueron procesados con el SPSS a fin de obtener frecuencias y correlaciones. Resultados: Se encontró que el manejo de la chacra es mayormente familiar, a excepción de la parte baja donde los campesinos si utilizan mano de obra pagada. El nivel educativo de los productores campesinos es bajo, no reciben capacitación del Estado o de otras instituciones privadas; una mejora importante del acceso a los servicios básicos; bajos ingresos provenientes de la actividad agropecuaria, observándose una marcada diferencia en los ingresos agropecuarios entre la parte alta y baja de la zona. Conclusiones: Existe una notoria diferencia entre la parte alta y baja en cuanto a sistemas de producción y nivel de especialización, lo cual se refleja en una evidente diferencia en los ingresos agropecuarios obtenidos, siendo muy bajos en la parte alta y mayores en la parte baja. Sin embargo, el acceso a los servicios básicos ha mejorado considerablemente en los últimos diez años.

Palabras clave: Producción agropecuaria tradicional, condiciones socioeconómicas, ingresos agropecuarios.

\section{ABSTRACT}

Objetive: Determine the predominant farming systems in the area and the socio-economic characteristics of population from Cordillera Negra in Carhuaz, Ancash. Methods: We applied surveys to a representative sample of 339 heads of family, of involved five districts: Yúngar, Anta, Marcará, Carhuaz y Ataquero; furthermore we also applied interviews to three community leaders and four mayors of districts in mention. The data obtained were processed with SPSS in order to obtain frequencies and correlations. Results: We found that the management of the farm is mostly family, with the exception of the low area, where the peasants normally used paid labor; that the educational level of the farmers is low, they receive no training from the State or other private institutions; we also found an important improvement of access to public basic services; little economic income from agricultural activities, noting a marked difference on agricultural revenues between the high and low zone. Conclusions: There is a noticeable difference between the high and low area referring to farming systems and level of specialization, which is reflected in a clear difference in the farming income, they are very little in the upper and some great at the low area. However, access to basic services has improved considerably in the last ten years.

Keywords: Farming systems, socioeconomic conditions, agricultural income.

\section{INTRODUCCIÓN}

En el Callejón de Huaylas, la agricultura tradicional sigue siendo la actividad más importante del ámbito rural, de ella depende un porcentaje importante de la población tanto rural como urbana, por que satisface las necesidades alimentarias de las familias campesinas y de las poblaciones de las principales ciudades de la zona. En este tipo de economías, la mitad a más de las cosechas obtenidas es producida por unidades domésticas campesinas, basándose principalmente en el trabajo familiar (Thorner, 1979). Por tanto, para los campesinos, sus unidades de producción son al mismo tiempo unidades de consumo, cuya finalidad es precisamente la reproducción de la familia o de la comunidad.

Debo mencionar que, la agricultura tradicional o familiar es una de las actividades que necesita mucha mano de obra y el uso preferente de aperos de labranza no mecánicos (Gografía rural, 2007), siendo un rasgo importante de la producción campesina el relativo alto grado de autosuficiencia. Las familias campesinas (las unidades de producción campesina) consumen una parte sustancial de su propia producción y, concomitantemente, producen casi todos los bienes que necesitan (Toledo, 2004). En el caso del Callejón de Huaylas, se puede afirmar que casi todas las familias campesinas alcanzan a cubrir las necesidades alimentarias, a partir de la actividad agropecuaria que ellas realizan, complementando, desde luego, con la recolección de especies de la flora y fauna silvestre que la naturaleza les brinda, como es el caso de la leña, plantas aromáticas, medicinales y la caza de aves. Así mismo, la mayor parte de los ingresos del campesino se deriva de la venta de la diversidad de productos agrícolas y pecuarios que ellos producen con su propio esfuerzo y dedicación, lo que implica a la vez que los medios de subsistencia son adquiridos, en cierta proporción, en el mercado.

En los trabajos de Chayanov y Marx (citados por E. Archette 1979), se menciona que la economía campesina típica presenta dos características comunes: El uso de la fuerza de trabajo familiar y la falta de acumulación de capital, debido a lo cual afirman que la economía campesina 
no sea típicamente capitalista, en tanto no se pueden determinar objetivamente los costos de producción por ausencia de la categoría salarios. Una característica importante de la producción tradicional de la zona es el apego a tecnologías tradicionales, que aseguran su persistencia. Justamente en relación a esto último, Mellor (1970) indica que tal actitud se debe fundamentalmente a un aspecto económico, que ve en el cambio de las formas de producción un alto riesgo y especialmente el alto costo que supone el fracaso. La adopción de nuevas técnicas significa en sí un rompimiento con la tradición, que es lo que ocurre en la parte baja del ámbito de estudio. Al respecto, Schultz (1965) indica que la agricultura tradicional se caracteriza por poseer un equilibrio interno, que la hace poco vulnerable al cambio.

Los sistemas de producción agropecuaria tradicional desarrollados en la zona, determinan las particularidades socioeconómicas de la población, que se describen es este trabajo y que pueden servir de línea base para futuros trabajos de investigación o para la toma de decisiones por parte de los dirigentes y autoridades de la zona, orientadas a mejorar la productividad y, con ello, las condiciones de vida de la población.

\section{MATERIALES Y MÉTODOS}

Tipo de investigación: La investigación realizada es básica, porque contribuye con cierto conocimiento referido a las características socioeconómicas de aquella población que depende de los sistemas de producción agropecuaria tradicionales, en la parte oriental de la Cordillera Negra en Carhuaz, Ancash. Tiene un enfoque mixto, es decir cuantitativo y cualitativo. La investigación se realizó a nivel descriptivo (deductivo); pero en algunos aspectos a nivel explicativo.

Diseño de investigación: En este caso fue un diseño no experimental, transversal a nivel descriptivo y explicativo, que consiste en conocer una realidad situacional o los valores que tienen una o más variables en el momento actual, describirlos y correlacionarlos en algunos casos (Hernández, et al, 2010). Se dice que es transversal por que se tomó la información de un momento único en el tiempo. Es descriptivo por que se reportan los datos tales como son en el tiempo y el espacio. Además es explicativo porque se describen algunas relaciones entre variables.

Población o universo: La población de estudio estuvo constituida por todas las familias que viven en el ámbito oriental de la Cordillera Negra (Callejón de Huaylas), en la provincia de Carhuaz, las mismas que están distribuidas en forma dispersa en cinco (05) distritos: Yungar, Anta, Marcará, Carhuaz y Ataquero, alcanzando un total de 2900 familias.

Unidad de análisis y muestra: La unidad de análisis estuvo constituida por una familia rural del ámbito de estudio, y la muestra por 339 familias que fueron encuestadas. Por tanto, se empleó como instrumento principal la encuesta que se aplicó a todas las familias integrantes de la muestra. También se hizo uso de la entrevista, que se aplicó a líderes y autoridades de los distritos localizados dentro del ámbito de estudio y también a algunos campesinos del lugar. Los datos obtenidos en campo fueron procesados empleando el SPSS, versión 21, con el cual se obtuvo promedios, correlaciones y el Chi cuadrado.

\section{RESULTADOS YDISCUSIÓN}

\section{Sistemas de producción}

Para lograr una evaluación objetiva, el ámbito de estudio se dividió en dos zonas de producción agrícola bien definidas: Parte baja y parte alta, cada una de las cuales presenta características topográficas y climáticas muy particulares, predominando determinados sistemas de producción agropecuaria en cada una de ellas.

[En la parte baja (entre los 2600 y $2900 \mathrm{msnm}$ ) predominan dos sistemas de producción a) frutales, alfalfa, cuyes y b) maíz, alfalfa, cuyes; sin embargo no necesariamente los tres componentes están presentes en los sistemas, pueden estar dos o tres de ellos]. En esta parte hay cierta tendencia a la especialización y a la producción en escala, condición que los diferencia radicalmente de la parte alta, donde no existe la especialización. Con esta estrategia los campesinos obtienen ingresos que les permite cubrir las necesidades de las familias. Esta condición me obligó a realizar la evaluación económica por separado; pero la caracterización social y ambiental en forma conjunta.

[En la parte alta (entre los 2900 y $3800 \mathrm{msnm}$ ) predominan los sistemas de producción a) papa (oca, olluco), trigo (cebada), habas (arveja), b) papa, maíz (trigo, cebada), arveja (habichuela) y cuyes; es decir, siempre incorporan en la rotación: tubérculos, cereales menores (o maíz), leguminosas, cuyes]. En algunos casos está presente también el ganado ovino 0 vacuno pero en cantidades relativamente pequeñas (vacunos 1 a 3 cabezas; ovinos entre 3 y 10 cabezas por familia). En esta parte la tendencia es tanto hacia los policultivos y la diversidad de crianzas, como estrategia para asegurar la alimentación de la familia, reducir los riesgos de pérdida de la producción y al mismo tiempo vender ciertos excedentes de productos agrícolas o pecuarios a fin de obtener algunos ingresos. El maíz también se incluye en la rotación, e incluso tiene preferencia frente a otros cereales, hasta una altura determinada ( $3300 \mathrm{msnm}$ ), más arriba este cultivo no prospera.

\section{Gestión de la chacra}

La agricultura tradicional se caracteriza por ser una empresa o actividad familiar, por eso en la mayoría de los casos de la zona, las chacras son manejadas estrictamente por la familia, especialmente en la parte alta.

Tabla 1. Tipo de mano de obra utilizada.

\begin{tabular}{ll}
\hline Tipo de mano de obra utilizada & $\%$ \\
\hline Familiar & 56 \\
Mano de obra pagada & 22 \\
Ambas & 22 \\
\hline
\end{tabular}

En la tabla 1 se puede ver que el $56,1 \%$ de las familias utiliza solamente mano de obra familiar; un $22,1 \%$ utiliza mano de obra pagada, aunque no en su totalidad (60\% de mano de obra pagada y $40 \%$ familiar) y $21,80 \%$ emplea ambas formas (60\% familiar y $40 \%$ pagada). Por tanto, en ningún momento el campesino utiliza $100 \%$ de mano de obra pagada, solo una parte de la mano de obra requerida y, esto ocurre cuando la parcela es relativamente grande o cuando la actividad a realizar requiere de mucha mano de obra, como es el caso de la cosecha de tuna, palta, la cosecha de tubérculos, pero también cuando el jefe del hogar realiza trabajos remunerados fuera de su localidad o tiene un trabajo permanente en el ámbito público o privado 
(alcaldes, regidores, albañiles, operarios, maestros de obra, técnicos, etc). Esto coincide con Thorner (1979), quien indica que en una economía campesina, la mitad o más de las cosechas es producida por unidades domésticas campesinas, basándose principalmente en el trabajo familiar. Otros afirman que en algunas zonas rurales (realidades de otros países), una alta proporción de los campesinos contrata sistemáticamente trabajadores asalariados, hasta el punto de que la participación del trabajo familiar puede ser menor que la de la mano de obra contratada (Lopera, 1998). Esto último se da en la parte baja del ámbito de estudio, donde la producción está en proceso de modernización.

Cabe mencionar que, el campesino conoce y calcula correctamente la mano de obra que necesita para realizar las diferentes labores culturales en sus campos, de acuerdo al tamaño de su chacra y a los cultivos sembrados. Remmers (1993) mencionaba que partiendo del hecho que la agricultura tradicional se ha desarrollado en estrecha relación con las necesidades de la sociedad a que atendía, por aquel entonces una sociedad que dependía mucho de la naturaleza, el manejo del medio ambiente se ha transformado en una manera de vivir de la población rural. Es ese conocimiento que convierte al campesino en un experto en manejar sus parcelas. Giddens (1979) afirma, que la fuerza del conocimiento tradicional de los agricultores deriva no sólo de las observaciones agudas, sino también del aprendizaje experimental.

\section{Grado de Instrucción de los productores}

Los resultados del estudio demuestran que un promedio de 6,0 a $6,5 \%$ de los encuestados es analfabeto; sin embargo al analizar el nivel de educación de los campesinos productores, se encontró una ligera diferencia entre la parte alta y baja.

Tabla 2. Grado de instrucción de la población campesina, parte alta y baja.

\begin{tabular}{lrlr}
\hline \multicolumn{4}{c}{ Grado de instrucción } \\
\hline Parte alta & $\%$ & Parte baja & $\%$ \\
\hline Analfabeto & 6,1 & Analfabeto & 6,5 \\
Primaria incompleta & 29,9 & Primaria incompleta & 17,4 \\
Primaria completa & 42,2 & Primaria completa & 26,8 \\
Secundaria incompleta & 14,3 & Secundaria incompleta & 24,6 \\
Secundaria completa & 6,1 & Secundaria completa & 21,8 \\
Superior & 1,4 & Superior & 2,9 \\
\hline
\end{tabular}

En la parte alta la gran mayoría (30 a 42\%) de los jefes de familia tiene primaria incompleta y completa y muy pocos llegan al nivel de secundaria incompleta y completa (14,3 y $6,1 \%$ respectivamente); en cambio los campesinos de la parte baja tienen mayoritariamente primaria completa y secundaria (incompleta y completa), e incluso hay un $2,9 \%$ que tiene educación superior (tecnológica y universitaria) (ver tabla 2). En cierta medida, la tecnología empleada y la forma de manejo de los cultivos y crianzas tienen estrecha relación con el grado de instrucción que tiene el productor. Cuanto menor es el nivel de educación mayor es el apego a la tradicionalidad del manejo y, viceversa, a mayor nivel de educación mayor tendencia hacia la modernidad y, por tanto, los rendimientos están en función del manejo y de la tecnología empleada.

\section{Acceso y calidad de la vivienda}

Los resultados de la tabla 3 demuestran que el $94,3 \%$ de las familias tienen casa propia y sólo un $5,7 \%$ no la tiene, estas últimas viven con los padres o en casa alquilada; se trata de matrimonios recientes o de familias que vienen de afuera y no han construido aún su propia casa.

Cabe mencionar que los campesinos, por lo general, tienen casa propia gracias a la existencia de una característica resaltante de las poblaciones rurales de la zona, se trata de la gran solidaridad entre familiares y vecinos. Después del matrimonio, la familia ayuda a los recién casados a construir sus casas, por su puesto con materiales de la zona, ya que la naturaleza les proporciona la mayoría de los materiales que se requieren para ello. Actualmente alrededor del $60 \%$ de las casas del ámbito rural (sobre todo de la parte alta) son de adobe, con cimientos de piedra, umbrales de madera y techo de teja; es decir con materiales que la naturaleza les brinda. Por eso, cada familia por lo general tiene su casa, grande o pequeña, perolatiene.

Tabla 3. Tenencia y calidad de las viviendas campesinas.

\begin{tabular}{lr}
\hline Tenencia y calidad de las viviendas & $\%$ \\
\hline Muy buena & 1,4 \\
Buena & 33,6 \\
Regular & 59,3 \\
No tiene & 5,7 \\
\hline
\end{tabular}

Además al recabar la percepción de los campesinos respecto a la condición en que se encuentra su vivienda, se encontró que el $59,3 \%$ de las familias considera que su casa está en condición regular; un 33,6\% dice que está buena y un $1,4 \%$ considera muy buena. Cabe recalcar que el concepto calidad en el mundo rural tiene una connotación relativa, por eso se ha preferido recabar la percepción de los mismos campesinos, en lugar de plantear parámetros citadinos para medir esa condición. Para algunos, por ejemplo, la casa es buena cuando es grande, para otros dependerá del material con que está construido, o de la ubicación, etc.

\section{Acceso a los servicios básicos}

Las investigaciones demuestran que en lo referente a servicios básicos, en los últimos 10 años, la situación, en el ámbito de estudio, ha mejorado considerablemente. Los resultados que se muestran en la tabla 4 indican que el $99 \%$ tiene acceso a la educación primaria; ese $1 \%$ que figura como que no tiene acceso, significa que la institución educativa de primaria se encuentra distante de su casa, alrededor de 40 min a más de una hora de camino, pero que en realidad pueden asistir haciendo un pequeño esfuerzo. En el caso de la educación secundaria la situación es diferente, se tienen que sólo un $47,9 \%$ de la población tiene acceso. Esto se debe a que, las instituciones educativas de secundaria se encuentran solamente en las capitales distritales y en dos centros poblados mayores (Poyor en Yungar y Tauripampa en Carhuaz), en los centros poblados menores no existen y, por eso, los jóvenes tienen que caminar diariamente entre 40 minutos a mas de una hora para llegar a los colegios secundarios. Probablemente esta situación sea una de las razones por las cuales la gran mayoría de los campesinos $(55,4 \%)$ solo tiene primaria (completa o incompleta) y no aspira a seguir estudiando.

En lo referente a agua potable, el $95,8 \%$ de las familias cuentan con ese servicio. Sin embargo, es necesario aclarar que en los diferentes centros poblados e incluso distritos, el acceso mas que al agua potable, es a 
una agua entubada, ya que en muchos lugares, el agua no recibe ningún tratamiento y en otros lo hacen en forma esporádica (de vez en cuando), lo importante aquí, es que las familias cuentan con agua apta para cocinar sus alimentos y otros usos, en su propia casa, ya sea en la parte interna o externa y no tienen que traerla desde las quebradas o ríos, como solía ser antes.

A la energía eléctrica tiene acceso el $95,5 \%$ de las familias, lo que indica un avance muy significativo y mejores condiciones de vida para las personas del ámbito rural. En lo referente al servicio de salud, el $76,9 \%$ de la población tiene acceso a una posta médica o a un centro de salud; el resto de la población tiene que desplazarse una hora de camino y en algunos casos un poco más para obtener el servicio.

Tabla 4. Acceso a los servicios básicos.

\begin{tabular}{cc}
\hline Acceso a los servicios básicos & Frecuencia \% \\
\hline Institución educativa primaria & 99,0 \\
Agua potable & 95,8 \\
Luz eléctrica & 95,5 \\
Posta médica & 76,9 \\
Teléfono & 52,8 \\
Institución educativa secundaria & 47,9 \\
\hline
\end{tabular}

El considerable logro del acceso a los servicios básicos se debe fundamentalmente al canon minero que recibe la población; en este caso recibe canon de la mina Barrick, Miskichilca y Antamina. Sin embargo, es necesario mencionar que para el logro de esta situación ha jugado un papel importante el Programa Foncodes (creado en el año de 1991 y comenzó a operar a partir de 1992), encargado de financiar proyectos de lucha contra la pobreza en el ámbito rural. Este programa fomentó y estimuló a los profesionales a diseñar y ejecutar proyectos en todo el ámbito del departamento, como una forma de crear su fuente de trabajo y obtener ingresos, hecho que contribuyó a la implementación de servicios básicos en casi todo el territorio.

\section{Ingresos agropecuarios de la población}

Para obtener la información referente a los ingresos de la población, se ha contabilizado tanto el monto proveniente de la venta de productos agrícolas y ganaderos, como también la cantidad, en forma valorizada, proveniente de los productos (animales y vegetales) destinados a la alimentación, manteniendo intangible un fondo familiar (como activo fijo), constituido por las semillas en el caso de los cultivos y el núcleo básico de animales que tiene cada familia, como punto de partida para trabajar, producir, vender y alimentarse. Cabe recordar también que todos los campesinos son tenedores precarios de medios de producción (tienen sus arados, herramientas, depósitos para productos, en algunos casos bueyes para el arado de los campos y burros o caballos para el transporte de sus productos); esos bienes no se contabilizan en los ingresos anuales.

Ingresos agropecuarios de los productores, parte baja

Teniendo en cuenta las consideraciones antes mencionadas, en la tabla 5 se exponen los ingresos agropecuarios de las familias de la parte baja (cerca a la rivera del río Santa), en donde la agricultura tradicional se encuentra en proceso de modernización. Se puede observar que hay una diferenciación muy grande en los ingresos, encontrándose familias cuyos ingresos provenientes de la actividad agropecuaria son mínimos (menos de 1000 o 2000 nuevos soles anuales) y otros que tienen ingresos considerables (mayores a 30,000 nuevos soles anuales), es decir que perciben ingresos mayores a los 2,500 nuevos soles mensuales. Si sumamos todos aquellos productores que tienen ingresos anuales mayores a 660 nuevos soles, el porcentaje llega a $31,5 \%$ de la población.

Tabla 5. Ingreso agropecuario anual de las familias, parte baja.

\begin{tabular}{cc}
\hline Ingresos agropecuarios en miles de nuevos soles & $\%$ \\
\hline < de 2 & 15,2 \\
de 2 a 6 & 37,7 \\
6 a 10 & 21,0 \\
10 a 20 & 13,8 \\
20 a 30 & 5,8 \\
30 a 40 & 6,5 \\
\hline
\end{tabular}

Cabe destacar que, en la parte baja, por un lado, las condiciones de clima son excelentes para una amplia gama de cultivos (temperatura promedio anual $16^{\circ} \mathrm{C}$ ), además cuentan con suficiente agua de riego y una carretera muy buena para el traslado de sus productos al mercado y, por otro, con la apertura de los mercados existe una mayor demanda de productos agropecuarios tanto para el mercado local, regional y nacional, como también para el mercado internacional. Esto ha provocado el interés de los campesinos de especializarse en algún cultivo o crianza, de tal manera que, en la actualidad, ya hay familias que se están dedicando exclusivamente a producir un determinado producto, es el caso de los productores de tuna, frutales (palta, lima, melocotón) o alfalfa en el caso de los cultivos y otros se están dedicando a la explotación de cuyes en forma masiva; es decir, se observa la paulatina especialización de los productores y, por tanto, una mejora en el nivel y calidad de vida de la población.

\section{Ingresos de los productores, parte alta}

En la parte alta (entre los 2900 y $3800 \mathrm{msnm}$ ), las condiciones son menos favorables para la producción agrícola, ya que por un lado, la gran mayoría de los terrenos son de secano y se encuentran en laderas con diferentes pendientes $y$, por otro, en esta zona se presentan con frecuencia ciertos fenómenos adversos como: Heladas, granizadas, sequías, etc., que influyen en forma considerable en el rendimiento de los cultivos. Esta condición obliga a los campesinos a utilizar estrategias para reducir el peligro de pérdida de las cosechas, una de ellas es justamente el uso de policultivos y de cultivos asociados. En la tabla 6 se puede observar que en la parte alta los ingresos anuales provenientes de la actividad agropecuaria son modestos, por ejemplo un $8,2 \%$ de las familias tienen ingresos inferiores a 1000 nuevos soles anuales (menores de 83,3 nuevos soles mensuales); el $41,5 \%$ obtiene ingresos que oscilan entre 1000 y 2000 nuevos soles; un $42,9 \%$ obtiene 2000 a 4000 nuevos soles y solo un $7,5 \%$ de las familias obtienen ingresos que oscilan entre 4000 y 6000 nuevos soles anuales.

Tabla 6. Ingreso Agropecuario anual de las familias de la Parte Alta.

\begin{tabular}{lr}
\hline Ingreso agropecuario, miles de nuevos soles & \multicolumn{1}{c}{$\%$} \\
\hline < de 1 & 8,2 \\
1 a 2 & 41,5 \\
2 a 4 & 42,8 \\
4 a 6 & 7,5 \\
\hline
\end{tabular}


Debido a los bajos ingresos obtenidos, muchos de los campesinos se ven obligados a vender su fuerza de trabajo para obtener ingresos complementarios que les permita satisfacer las necesidades básicas de vestido, alimentación, vivienda, etc. Algunos trabajan temporalmente en la parte baja o en las ciudades del Callejón de Huaylas; otros son albañiles o maestros de obra y migran con frecuencia a otros lugares a trabajar; pero también hay guías turísticos, comerciantes, etc. Esto concuerda con Toledo (2004), quien menciona que la subsistencia campesina está basada en una combinación de actividades, que incluyen la recolección agrícola, cuidado de ganando doméstico, artesanía, pesca, caza y trabajos fuera de la explotación a tiempo parcial, estacionales o intermitentes.

\section{Ingresos complementarios comparativos, parte alta y baja}

Muchos de los campesinos productores no obtienen los ingresos agropecuarios suficientes que les permita satisfacer las necesidades básicas de él y de su familia, viéndose obligados a buscar ingresos complementarios, que pueden ser en base a la venta de su fuerza de trabajo o producto de otras actividades no agropecuarias, como el comercio, venta de servicios (acompañante de turistas, alquiler de molino de granos, alquiler de sierra, etc), venta de artesanías, albañilería, maestro de obras, regidor, profesor, técnico, etc.

Los datos de la tabla 7 demuestran, por un lado, que tanto los campesinos de la parte alta como baja realizan trabajos no agropecuarios, por los cuales obtienen ingresos complementarios y, por otro, que hay ciertas diferencias en cuanto a la cantidad de ingresos que obtienen.

Tabla 7. Ingresos no agropecuarios de la parte alta y baja.

\begin{tabular}{lclr}
\hline \multicolumn{3}{c}{ Ingresos no agropecuarios de la parte Alta y } & Baja \\
\hline Parte alta & $\%$ & Parte baja & $\%$ \\
\hline Ninguno & 29,3 & Ninguno & 31,2 \\
< de 2 & 49,7 & < de 2 & 19,6 \\
De 2 a 6 & 13,6 & De 2 a 6 & 32,6 \\
De 6 a 10 & 4,1 & De 6 a 10 & 7,2 \\
De 10 a 20 & 0,7 & De 10 a 20 & 8,7 \\
De 20 a 30 & 1,2 & De 20 a 30 & 0,7 \\
De 30 a 40 & 1,4 & De 30 a 40 & 0,0 \\
\hline
\end{tabular}

En primer lugar, el porcentaje de campesinos de la parte alta y baja, que no realizan otras actividades, que no sean las agropecuarias, alcanzan 29,3 y $31,2 \%$ respectivamente; en segundo lugar, un alto porcentaje de campesinos de la parte alta reciben menos ingresos complementarios que los de la parte baja, lo cual probablemente esté relacionado con el tipo de trabajo que realizan y también del lugar donde lo ejecutan, por ejemplo hay maestros de obra u obreros de las minas que reciben salarios mucho mayores que otros, que trabajan como albañiles o como peones en los campos de cultivo.

\section{DISCUSIÓN}

Las condiciones socioeconómicas de la población de la zona oriental de la Cordillera Negra, en Carhuaz, Ancash, están relacionadas con la disponibilidad de capital natural (suelo y agua), con los sistemas de producción emprendidos y con el nivel de especialización de los productores.
En la población campesina predomina el nivel de educación primaria (incompleta y completa) y la secundaria incompleta, observándose que cuanto menor es el nivel de educación del productor, mayor apego tiene hacia la tradicionalidad y, viceversa, a mayor nivel de educación, mayor es la tendencia hacia la modernidad y la especialización.

Se encontró que, en los últimos 10 años ha mejorado considerablemente la situación social; más del $95 \%$ de la población cuenta con agua y energía eléctrica en sus hogares; el 99\% tiene acceso a la educación primaria y un $47,9 \%$ a la educación secundaria. Así mismo, el 76,9\% tiene acceso a una posta médica o centro de salud. Sin embargo, esta situación no es el resultado de la actividad agropecuaria, sino del canon minero del cual son beneficiarios.

Entre la parte alta y baja del ámbito de estudio, existe una marcada diferencia en los ingresos agropecuarios de los productores, es significativamente mayor en la parte baja, favorecidos por las ventajas competitivas y los sistemas de producción emprendidos por la población.

\section{REFERENCIAS BIBLIOGRÁFICAS}

Archetti, E. (1979). Economía Campesina: Presentación a Chayanov, la Organización de la Unidad Económica Campesina. Lima: Desco.

Geografía rural. (2007). Los sistemas agrarios tradicionales (en línea). Consultado el 12 de Noviembre del 2012. Disponible en: http://geografia.laguia2007.com/geografiarural/sistemas-agrarios-tradicionales.

Giddens, A. (1979). Central problems in social theory: action, structure and contradiction in social analysis. London. Macmillan.

Hernández, S; Fernández, C y Baptista, L. (2010). Metodología de la Investigación. México: Ed Mc Graw Hill/Interamericana Editores S.A. Quinta edición.

Lopera P, J. (1998). Corporación Colombiana de Investigación Agropecuaria, Corprica. Lecturas sobre Economía campesina y Desarrollo Tecnológico (en línea). Consultado en Enero 2013. Disponible en: h t t p : / / w w w.g e o c i t i e s.com / SiliconValley/Way/4302/economia1.

Mellor, J., (1970). The Subsistence Farmer in Traditional Economic, en C. Wharton (ed.): Subsistence Agriculture and Economic Development, London, Frank Cass, p. 217.

Montalba N, R. (2004). Cambio Técnico Agrario y la industrialización de la Agricultura: Características, motivaciones y efectos. Reflexiones vía Internet del Dr. Montalba, profesor de Agroecología Fac. Cs. Agropecuarias, U. de La Frontera.

Remmers , G. (1993). Agricultura tradicional y agricultura ecológica: vecinos distantes. Instituto de Sociología y Estudios Campesinos. Universidad de Córdoba. Rev. Agricultura y Sociedad No 66. Pp 201-220

Schultz, T. (1965). Transforming Traditional Agriculture, New Haven on London, Yale University Press, p. 30.

Toledo V.M. (2004). La Racionalidad Ecológica de la Producción Campesina. Universidad Nacional de México, (En línea), consultado el 12 de Enero 2015 en http://www.clades.cl/revistas/5/rev5art3.htm.

Thorner, D. (1979). La Economía Campesina, concepto para la Historia económica. En Economía campesina. Lima: Desco. 